\title{
A binary population synthesis study on gravitational wave sources
}

\author{
Liu, Jinzhong and Zhang, Yu \\ Xinjiang Astronomical Observatory, Chinese Academy of Sciences, 150 Science 1-street \\ Urumqi, Xinjiang 830011, China \\ email: liujinzh@xao.ac.cn
}

\begin{abstract}
Gravitational waves (GW) are a natural consequence of Einstein's theory of gravity (general relativity), and minute distortions of space-time. Gravitational Wave Astronomy is an emerging branch of observational astronomy which aims to use GWs to collect observational data about objects such as neutron stars and black holes, about events such as supernovae and about the early universe shortly after the big bang. This field will evolve to become an established component of 21st century multi-messenger astronomy, and will stand shoulder-to-shoulder with gamma-ray, x-ray, optical, infrared and radio astronomers in exploring the cosmos. In this paper, we state a recent theoretical study on GW sources, and present the results of our studies on the field using a binary population synthesis (BPS) approach, which was designed to investigate the formation of many interesting binary-related objects, including close double white dwarfs, AM CVn stars, ultra-compact X-ray binaries(UCXBs), double neutron stars, double stellar black holes. Here we report how BPS can be used to determine the GW radiation from double compact objects.
\end{abstract}

Keywords. gravitational waves, binaries, stellar content.

\section{Introduction}

The Laser Interferometer Gravitational-Wave Observatory or LIGO, a joint project between Massachusetts Institute of Technology (MIT) and California Institute of Technology (Caltech) is spearheading with equally ambitious projects such as VIRGO, TAMA 300 and GEO 600 (Tagoshi et al. 2001 and references therein). Specially, the eLISA is a planned space based GW observatory at low frequency with the launch date in the 2018+ time frame (Amaro-Seoane et al. 2012 and references therein). The different frequency ranges of the GW detectors will respectively correspond to different observation sources. The main purpose of this paper is to explore the different GW sources with respect to the ground or space detectors. Specially, we focus on the compact binaries as GW sources. For instance, we used population synthesis models to create a population of compact binary (Liu et al. 2012) and discussed the effect of these binaries as both foreground noise and resolvable sources for the eLISA GW detector (Liu 2009, Liu et al. 2010a, 2010b, Liu et al. 2014).

\section{Computations}

To systematically investigate the GW radiation from different GW sources we performed a Monte Carlo simulation through the evolution of a sample of 1 million binaries. The description of our input physics parameters is given as follows. 1) The properties of GW sources, which can be derived from some observations, provide some basic information. 2) A star formation rate is necessary in the study. 3) Mass function with different redshift is another important case. 4) GW sources will be disappeared within Hubble 


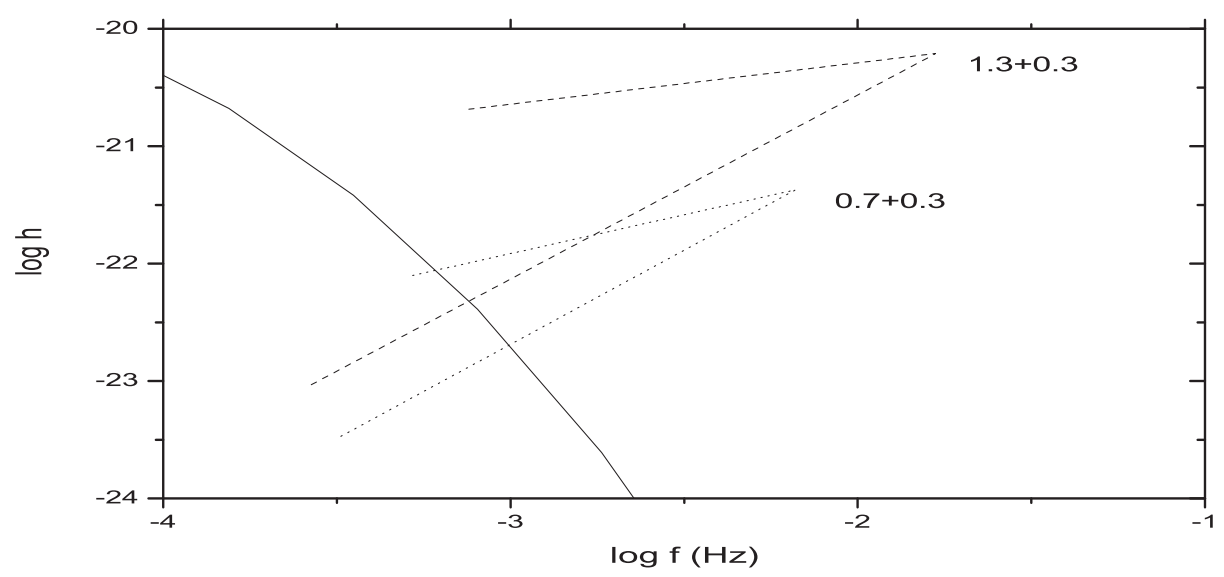

Figure 1. Evolutionary trace of AM CVn stars (NS+He WD) and UCXBs (CO WD+He WD) within Hubble time. The solid line stands for the foreground floor coming from CDWDs.

time, a factor of birth or merger rate maybe influence the formation of GW sources. 5) To construct the GW systems, other typical parameters in this work are need to be displayed, such as the velocity dispersion, Luminosity relation, space distribution in the Galaxy or different redshift, eccentricity and the cosmological parameters.

\section{Results and discussion}

Mass transfer binaries with orbital periods less than 1 hour are driven by gravitationalwave radiation, such as AM CVn systems and ultra-compact X-ray binaries (UCXBs). Figure 1 gives an evolution process of AM CVn and UCXB from detach state to mass transfer state. We can see that due to owning a more mass compact component the GW radiation from UCXBs is stronger than AM CVn. These two GW sources can be resolved by space telescope (e.g. eLISA), because its amplitude is higher than foreground floor (see the solid line). So the mass-transfer binaries with less periods are thought to be the primary astrophysical sources of GW for space detector. Note that when these binaries during the merger process in the end of binary evolution will give a main contribution within the frequency band of ground-based detectors. Finally, these GW sources can be observed using optical, X-ray, and radio telescopes.

\section{Acknowledgements}

This work is supported by the program of light in China's Western Region (LCWR) (No. XBBS201221 and XBBS201022), the Xinjiang Natural Science Foundation (No. 2011211A104), and the Natural Science Foundation (No. 11103054 and 11303080).

\section{References}

Amaro-Seoane, P., et al. 2012, Classical Quant. Grav., 29, 124016

Liu, J. Z. 2009, MNRAS, 400, 1850

Liu, J. Z., Han, Z., Zhang, F., \& Zhang, Y. 2010a, ApJ, 719, 1546

Liu, J. Z., Zhang, Y., Han, Z., \& Zhang, F. 2010b, Ap\&SS, 329, 297

Liu, J., Zhang, Y., Zhang, H., Sun, Y., \& Wang, N. 2012, A\&A, 540, 67

Liu, J. Z. \& Zhang, Y. 2012, PASP, 126, 211

Tagoshi, H., Kanda, N., Tanaka, T., et al. 2001, Phys. Rev. D., 32, 062001 